\title{
Structural and functional types of lexical units, their expressiveness
}

\section{[Types structurels et fonctionnels des unités lexicales, leur expressivité]}

\author{
Kulyash Duisekova - Saule Issabekova - Aliya Zagidullina - Gulshat \\ Beysembayeva - Aitkali Bakitov - Peter Ziak
}

DOI: $10.18355 / X L .2021 .14 .04 .17$

\begin{abstract}
At the present stage of development of linguistic science and in context of global trends towards constant cooperation in various fields and spheres of activity, there is a need for comparative typological studies aimed at a comprehensive and large-scale study of various linguistic categories. First of all, this is explained by the fact that it is in these categories, as in a mirror, that the peculiarities of the mentality, history and secular culture of the speakers of a particular language are reflected.

The object of language sciences consists of two series of phenomena: on the one hand, it is made up of everything that is inherent in human language, that is to say language as such in its relation to thought and to reality; on the other hand, we are talking about the specifics and the variety of properties and categories of each language compared to another. Researchers' attention has largely shifted from the question of how the language of speakers of a particular language works.

Research plays an important role in the study of the specificities of the nominative means of the language. Contrastive linguistics has the great advantage that the practical areas of its application are clearly visible. These are mainly the methodology and methodology of foreign language teaching, bilingual lexicography, theory and practice of translation.
\end{abstract}

Key words: structural and functional types, phraseological units, French, expressiveness

\section{Résumé}

Au stade actuel du développement de la science linguistique et dans le contexte des tendances mondiales vers une coopération constante dans divers domaines et sphères d'activité, il existe un besoin d'études typologiques comparatives visant à une étude complète et à grande échelle de diverses catégories linguistiques. Tout d'abord, cela s'explique par le fait que c'est dans ces catégories, comme dans un miroir, que se reflètent les particularités de la mentalité, de l'histoire et de la culture séculaire des locuteurs d'une langue particulière.

L'objet de sciences du langage consiste en deux séries de phénomènes : d'une part, il se compose de tout ce qui est inhérent au langage humain, c'est-à-dire le langage comme tel dans son rapport à la pensée et à la réalité ; d'autre part, nous parlons des spécificités et de la variété des propriétés et des catégories de chaque langue par rapport à une autre. L'attention des chercheurs s'est largement déplacée de la question du fonctionnement du langage des locuteurs d'une langue particulière.

La recherche joue un rôle important dans l'étude des spécificités des moyens nominatifs de la langue. La linguistique contrastive a le grand avantage que les domaines pratiques de son application sont clairement visibles. Il s'agit principalement de la méthodologie et de la méthodologie de l'enseignement des langues étrangères, de la lexicographie bilingue, de la théorie et de la pratique de la traduction.

Mots clés : types structurels et fonctionnels, unités phraséologiques, français, expressivité 


\section{Introduction}

L'aspect comparatif de l'étude systémique de la phraséologie est sans aucun doute d'un grand intérêt tant pour l'élaboration d'une théorie générale de la phraséologie que pour l'étude des traits communs et distinctifs des langues étudiées. L'objet de notre recherche est les unités phraséologiques-somatismes dans la langue française.

Même dans les temps anciens, une personne mesurait le monde qui l'entourait avec des parties de son corps, en particulier avec sa tête, ses bras et ses jambes. En utilisant leurs noms au sens figuré, les gens expriment plus pleinement leurs pensées et leurs sentiments. Par conséquent, les noms des parties du corps sont un objet d'étude important en linguistique moderne. Ces unités forment un sous-système phraséologique et couvrent les sphères de corrélation conceptuelle générale. Dans les langues comparées, leur analyse révèle à la fois des traits communs inhérents à la communauté humaine, quelle que soit l'appartenance linguistique, et des traits spécifiques dus à des facteurs extralinguistiques, notamment nationaux.

Le mot phraséologie a plusieurs sens. En tant que terme linguistique, il est utilisé pour désigner une branche spéciale de la linguistique qui étudie des phrases stables appelées unités phraséologiques (unités phraséologiques), moins souvent des phrases phraséologiques, ainsi que pour désigner un ensemble de phrases similaires caractéristiques d'une langue particulière.

Le dictionnaire encyclopédique linguistique édité par VN Yartseva contient la définition suivante de l'unité phraséologique : « lois du choix et combinaisons de mots lors de l'organisation d'un énoncé, mais qui sont reproduites dans la parole dans un rapport fixe de la structure sémantique et d'une certaine composition lexicale et grammaticale » (LES, 1990).

Dans le même dictionnaire V.N. Telia écrit que le terme unité phraséologique désigne plusieurs types de combinaisons sémantiquement hétérogènes : 1) des idiomes caractérisés par une refonte de leur composition lexicale et grammaticale et possédant une fonction nominative intégrale (laver le linge sale en public, à la poursuite, corbeau blanc), ils sont juxtaposés par des combinaisons de phrases, dans lesquelles la structure syntaxique et une certaine partie de la composition lexicale sont repensées, et le reste est rempli dans le contexte (quel que soit le jour (heure, année...), alors..., pour les pères (fils, filles...) convient ; 2) les combinaisons, dans lesquelles un seul mot est lexicalement repensé tout en conservant une fonction nominative distincte pour chacun des mots composants (entrer en conflit, entrer dans la conversation) ; 3) les tampons vocaux tels que demander des mots, comment allez-vous, etc.; sont proches d'eux ; 4) proverbes et dictons formés dans le folklore (ne vous asseyez pas dans votre traîneau); 5) mots ailés - dictons de nature aphoristique, remontant à un certain auteur ou à une source littéraire anonyme (Homme - ça sonne fièrement! Etc.). Les chercheurs en composition phraséologique ont de nombreuses opinions sur ce qu'est une unité phraséologique, et il existe une différence de points de vue sur la composition de ces unités dans chacune des langues. Ainsi, par exemple, N.M. Shansky inclut des proverbes, des dictons, des mots ailés, des aphorismes en unités phraséologiques (Shansky, 1969). Le dictionnaire phraséologique (FS), dans les unités phraséologiques, comprend divers tournures de discours descriptives et analytiques, des conjonctions complexes, des prépositions complexes, des termes composés, etc. En général, une unité phraséologique est caractérisée comme «une combinaison de mots avec un sens figuré», comme une «phrase stable avec un sens idiomatique», comme une "phrase stable». Les phraséologismes sont caractérisés par la métaphore, l'imagerie, la coloration expressive et émotionnelle.

N.M. Shansky pense que les phrases phraséologiques incluent à la fois des combinaisons stables de mots, sémantiquement équivalentes à un mot, et l'unité, 
structurellement et sémantiquement, représentant des phrases, c'est-à-dire littéralement toutes les unités reproduites sous une forme finie (Shansky, 1969).

La tournure phraséologique diffère principalement de la phrase libre par la généralisation du sens de l'ensemble de la tournure dans son ensemble. C'est ce qui permet de mettre en évidence un type particulier de sens du retournement - sens phraséologique, qui ne coïncide pas avec le sens lexical des mots - les composants qui le composent.

De plus, le sens phraséologique, en règle générale, n'est pas direct, mais figuratif, résultant de sens primaires et nominatifs de mots dans certaines combinaisons libres.

E.F. Arsentieva soutient que les unités phraséologiques sont un caillot d'informations culturelles et permettent de dire beaucoup, en économisant les moyens linguistiques, en pénétrant les profondeurs de l'esprit national, de la culture (Arsentieva, 1993). Elle pense que l'existence d'éléments communs et internationaux est observée dans la sémantique des unités phraséologiques des langues russe et française, ce qui est dû à divers facteurs linguistiques.

Selon E.F. Arsentieva, des éléments internationaux dans la sémantique des unités phraséologiques apparaissent à la suite d'un parallélisme phraséologique, qui repose sur la même refonte des phrases libres originales. Les tropes jouent un rôle important dans le développement du parallélisme phraséologique. La refonte de combinaisons de mots variables dans différentes langues passe par un transfert métaphorique, métonymique ou figuratif séparé ou cumulatif. Par exemple, entêté (têtu) comme une souche - têtu comme un âne; trembler du froid - trembler comme une feuille; gratteciel (Arsentieva, 1993).

Yu. Solodub soutient également que le fonds phraséologique de la langue peut contenir des unités phraséologiques internationales, des unités phraséologiques nationales propres et des unités phraséologiques mixtes (Solodub, 1985). Selon lui, l'identité nationale et les éléments internationaux dans le système de phraséologie d'une langue particulière sont interconnectés. Il croit que toute éducation phraséologique internationale contient des caractéristiques et des qualités nationales. D'autre part, dans le national, il n'y a pas seulement un trait particulier qui distingue la phraséologie d'une langue donnée, mais aussi un trait général qui est caractéristique de toutes les langues. Il ne peut pas y avoir d'unités phraséologiques purement internationales et purement nationales dans un système phraséologique. Par conséquent, en phraséologie, il considère les éléments généraux et spécifiques comme des contraires dialectiques existant dans l'unité. Il affirme que cette unité s'exprime dans l'interpénétration, dans la transition l'une dans l'autre. En termes d'expression, les unités phraséologiques peuvent appartenir à l'une ou l'autre langue nationale, et en termes de contenu, elles sont la propriété de la culture et de la civilisation mondiales.

Selon L.I. Roizenson, les somatismes ont une forme interne, c'est-à-dire le lien qui existe entre le sens phraséologique du renouvellement et le sens réel de ses composants (Roizenson, 1965). Il soutient que pour de nombreuses unités phraséologiques avec une composante somatique, la forme interne est constituée de divers mouvements corporels, par exemple : gestes, démarche, expressions faciales, posture ; le bien-être d'une personne ; processus physiologiques par lesquels les actes de la vue, de la parole, de la perception auditive, de la prise alimentaire, du mouvement, de l'activité physique sont effectués; processus d'impact physique sur une personne; l'apparence d'une personne; certaines actions d'une personne selon la situation.

N.N. Kirillova conclut que la composante somatique, étant un nom, a les catégories grammaticales suivantes : la catégorie du nombre et la catégorie de la certitude/indétermination (Kirillova, 1975). Selon Kirillova, dans le groupe des 
somatismes, chaque nom a séparément son propre ensemble de sèmes et leurs distributions, qui ne se répètent pas autrement dit de la même série : doigt et dos, etc. Et, ils sont complètement incommensurables dans tout le reste, à l'exception de leur appartenance à un être vivant.

Pour la première fois, le terme "somatique » a été introduit dans l'usage linguistique dans les études finno-ougriennes par F. Vakk, qui, considérant les unités phraséologiques de la langue estonienne qui contiennent les noms des parties du corps humain en mots, les a appelés somatiques. Il conclut qu'ils sont l'une des couches les plus anciennes de la phraséologie et constituent la partie la plus commune de la composition phraséologique de la langue estonienne (Vakk, 1968).

Le terme "unité phraséologique somatique » appliqué au matériau de la langue a été utilisé pour la première fois par EM Mordkovich dans l'article «Groupes sémantiques-thématiques d'unités phraséologiques somatiques ", où il affirme que la plupart des unités phraséologiques somatiques agissent comme une caractéristique négative (Mordkovich, 1971).

Les phraséologismes-somatismes sont le matériau principal de notre recherche. Il existe un assez grand nombre d'ouvrages sur la phraséologie somatique.

\section{Méthodes}

Dans le cadre de la méthode comparative linguistique générale, les méthodes et techniques de recherche particulières suivantes sont utilisées dans le travail: (1) analyse contrastive - pour identifier les similitudes et les différences dans la forme et le contenu des unités phraséologiques-somatismes de la langue française ; (2) analyse des composants - pour identifier les équivalents complets, partiels et non équivalents ; les significations de ces derniers sont révélées au moyen de la traduction ; (3) traitement tabulaire et calcul quantitatif - pour établir la productivité des groupes lexico-sémantiques des unités phraséologiques-somatismes comparés.

\section{Discussion}

Les somatismes avec une composante tête / tête symbolisent l'activité mentale d'une personne, pensant en général à des degrés et degrés divers. Selon GG Sokolova, en français il y a 328 somatismes avec une composante tête, en russe il y a 225 somatismes avec une composante tête, donc, en français il y a 103 somatismes avec une composante tête de plus qu'en russe (Sokolova, 2001).

L'irradiation métaphorique des synonymes est très caractéristique de la langue française. Après un mot, d'autres mots du même groupe lexico-sémantique commencent à acquérir un sens figuré similaire. Ainsi, en russe, pour désigner la « tête » en langage familier, on utilise des noms métaphoriques pour les plats : un chapeau melon ou, moins souvent, une cruche. En français vernaculaire, une rangée plus longue de mots du même champ sémantique est utilisée pour cela : carafe, carafon, cafetière, bouilloire, tirelire. La tête peut être désignée dans le langage courant non seulement par le mot poire, mais par le nom de presque tous les légumes ou fruits : chou, cédrat, cale-basse, coloquinte, coco, ciboulot et même cassis. La facilité d'un même type de transfert de mots d'un même groupe lexico-sémantique indique une moindre clarté des frontières sémantiques entre les membres de ce groupe.

Dans la conscience mythologique, l'homme est le centre de l'univers. Les anciens voyaient en lui une incarnation antromorphique de l'Univers, car ils associaient la position verticale qu'il occupait à l'effort vers le ciel, à des pensées «élevées ». L'horizontale chez l'homme signifiait pour eux tout ce qui était terrestre, périssable. La tête est le centre du traitement de l'information, chez l'homme ancien, tout ce qui est lié à la tête était en corrélation avec le ciel et ses principaux objets - le soleil, la 
lune, les étoiles. Le mythologème de la tête (dans notre compréhension, le mythologème est un emprunt conscient de motifs mythologiques et leur transfert dans le monde de la culture artistique moderne) - «le soleil» - a constitué la base d'unités phraséologiques telles que la tête tourne, le la tête est en feu, la tête tourne.

Un autre mythologème de la tête - "Dieu, l'essentiel, l'important" - se reflète dans les unités phraséologiques de la tête (à propos de l'important), la tête d'or (à propos d'une personne intelligente).

La majeure partie des unités phraséologiques russes avec la composante principale a été formée plus tard et a perdu le lien avec les mythologèmes mentionnés. Or ces unités phraséologiques désignent principalement les capacités intellectuelles d'une personne, ses qualités, ses conditions physiques, etc. Par exemple, la tête fuit, la tête est en place, la tête cuisine - à propos d'une personne intelligente; sans roi dans sa tête, une tête verte, une tête de poulet, une tête de chêne, une tête de jardin - à propos d'une personne stupide et bornée.

Désignant la partie la plus importante d'une personne, le mot tête forme des unités phraséologiques qui caractérisent une personne sous divers angles : comme de la neige sur la tête (de façon inattendue), même un pieu sur la tête (à propos d'une personne têtue), une tête inflexible ( sur une personne rebelle), la tête gonfle (l'état d'une personne), une tête invétérée (sur une personne désespérée), une tête brûlante (sur une personne ardente, sur une personne qui a une tête petite), une tête médiocre (sur une personne malheureuse), etc. Selon V.G. Gak, les mots qui ont un sens dénotatif commun (indiquant le même objet) peuvent avoir différentes nuances de sens connotatif, car dans leur sens ils peuvent refléter différents aspects de l'objet désigné (Gak, 1977). Les nuances connotatives se manifestent principalement dans le sens figuré et phraséologique des mots. Ainsi, les mots tête et tête désignent la même partie du corps, mais le mot français désigne souvent un visage, et s'avère souvent être synonyme des mots visage ou mine, comme en témoignent les combinaisons : tête sympatique, avoir une tête comique / une drôle de tête etc.

Étant donné que le mot tête est souvent utilisé dans le sens de "visage, expression faciale", le mot grue est parfois utilisé pour désigner la tête réelle - un crâne, par exemple, dans l'expression // une grue grand-de-taille - il a un mal de tête.

Sur la base de l'analyse sémantique des somatismes à cette composante, on distingue les groupes lexico-sémantiques communs aux langues étudiées :

1) caractère,

2) condition physique,

3) sentiment-état,

4) sentiment-attitude,

5) mental activité,

6) portrait,

7 ) actions et actes d'une personne,

8) statut social (Aprisyan, 2006).

Le groupe lexico-sémantique des unités phraséologiques somatiques françaises à composante des yeux est l'un des plus productifs dans la formation des somatismes. Selon GG Sokolova, les unités phraséologiques avec une composante œil / œil se classent au troisième rang - en français avec une composition quantitative de 263 unités. Les yeux sont la partie la plus importante de la tête et du visage humains. Dans les complexes verbaux stables russes, en plus de la composante des yeux, le mot vieux slave oko fonctionne - au singulier. L'heures ou les yeux - au pl. h., qui jusqu'au XVIème siècle a été utilisé comme d'habitude, généralement accepté, stylistiquement neutre et au $18^{\text {ème }}$ siècle fait partie des mots de grand style. Le mot œil désigne l'organe de vision de tout être vivant. Les yeux sont les yeux d'une personne sont - de 
plus - beaux, grands, expressifs. Ce sont les yeux qui caractérisent dans la langue russe non seulement les capacités physiques, mais aussi spirituelles d'une personne à comprendre les phénomènes, c'est-à-dire la vision intérieure. Ils sont l'organe de l'intuition : voir avec les yeux mentaux, voir avec l'œil intérieur, les yeux de l'âme, les yeux du cœur, les yeux spirituels.

La langue marque avec précision la capacité inhabituelle des yeux - leurs pupilles sont en mouvement, d'où la compatibilité d'un grand cercle de verbes de mouvement avec le mot "yeux » : regardez autour de vous avec les yeux, détournez les yeux, faites glisser vos yeux, mesure tes yeux, cherche tes yeux, suis tes yeux, rive tes yeux, etc. Les yeux sont un instrument-orgue, un organe du «regarder». Par conséquent, nous restons bouche bée de surprise et de surprise, nos yeux s'ouvrent grand lorsque nous cherchons inconsciemment à recevoir un maximum d'informations à travers eux, nous plissons les yeux lors d'une observation rapprochée ou avec une forte concentration de pensée, détournons les yeux sous le regard condamnant de quelqu'un, protégeant ainsi notre cerveau de l'impact négatif de l'interlocuteur, etc.

Le soleil et la lune dans les mythologies de nombreux peuples étaient considérés comme les yeux d'une puissante divinité. A ce mythologème sont associées des unités phraséologiques de l'œil du maître (supervision fiable sur quelque chose), sans œil (sans supervision).

Un autre mythologème est un œil = une personne, qui a donné lieu à de nombreuses unités phraséologiques : l'œil est planifié (à propos d'une personne expérimentée), l'œil se repose (à propos d'une impression visuelle agréable), l'œil se réjouit (à propos d'un événement joyeux qui peut être vu), les yeux sont trompeurs (à propos de ceux qui doutent de la fiabilité de ce qu'ils ont vu), les yeux s'illuminent (à propos d'un fort désir chez une personne); des métaphores telles que les yeux parlent, les yeux courent, les yeux ont honte ; dictons yeux envieux, saisissant les mains (sur l'insatiabilité de la nature humaine), etc. Puisque $80 \%$ des informations sur le monde passent par les yeux, ils sont considérés comme les organes les plus importants, on leur attribue un mystérieux pouvoir magique. Dans les unités phraséologiques avec la composante "yeux", d'anciens stéréotypes de comportement ont été fixés et préservés à ce jour vous ne pouvez pas quitter les yeux (c'est ainsi que vous deviez communiquer avec l'interlocuteur), pour détourner vos yeux et les autres.

Tromper quelqu'un, c'est interférer avec la perception adéquate du monde, c'est-à-dire d'abord interférer avec lui pour regarder, d'où les unités phraséologiques pour couvrir les yeux, jeter de la poussière dans les yeux.

Depuis l'Antiquité, les amulettes, fabriquées à partir de métaux et de pierres précieuses et en forme d'œil, ont été remontées du mauvais œil, d'où les unités phraséologiques du type œil-diamant (sur la capacité de voir important, basique), prendre soin comme un œil (faire très attention), prendre ses yeux dans ses mains (faire attention), avec l'oeil armé (la forme moderne de cette unité phraséologique à l'oeil nu), etc.

Il existe huit groupes lexicaux et sémantiques communs aux langues étudiées : 1) caractère, 2) condition physique, 3) état émotionnel, 4) état émotionnel, 5) caractéristiques qualitatives d'une personne, 6) activité mentale, 7) portrait , 8 ) les actions et les actes d'une personne (Mordkovitch, 1974).

1. Caractère : Le groupe lexico-sémantique "Caractère » est représenté par un caractère négatif dans la langue française.

Les caractéristiques négatives comprennent : 1) l'importunité, 2) l'arrogance, 3) la duplicité.

1) importunité : fr. lang.: crever les yeux, cracker dans les yeux rus. lang.: piquer dans les yeux, monter dans les yeux, callou les yeux, il n'y a pas de honte dans les

XLinguae, Volume 14 Issue 4, October 2021, ISSN 1337-8384, eISSN 2453-711X 
yeux, filer devant les yeux, jeter dans les yeux, poudre bleue dans l'oeil; 2) arrogance : f. langue: mesurer les (avec les) yeux rus. laz.: mesurer avec les yeux; 3 ) duplicité derrière les yeux. 2. Le groupe « État physique » comprend les somatismes exprimant les états suivants : 1) la capacité de voir, 2) la somnolence, 3) le sommeil,) 4) la douleur. Français, 1) la capacité de voir : fr. langue: jeter un coup d'œil, aux yeux de qn rus. laz. : même si tu t'arraches les yeux, au moins d'un œil, pour apparaître dans tes yeux, mordre avec tes yeux, devant tes yeux, regarder avec tes yeux, avec tes propres yeux, où que tu regardes, disparaitre de tes yeux, ne pas voir dans tes yeux (ne pas voir);

Dans chacun de ces traits de la langue française, les somatismes jouent un rôle actif, et dans ces derniers, les noms de parties du corps détiennent leur monopole, puisque tout au long de l'histoire de la langue française, de la rhétorique de la Renaissance aux grammairiens modernes, la langue française remplit « cette profusion de bras, de mains, d'yeux, de pieds et d'autres membres du corps qui remplacent à chaque instant les personnages à qui ces membres appartiennent » (Wey, 1963).

Selon V.G. Gak, cette technique existe comme une recommandation pour remplacer les mots homme, gens, personnes par les noms de parties du corps œil, bras, main, tête,cœur (Gak, 1959).

Le groupe lexico-sémantique des somatismes de langue française contient plus de 100 lexèmes, dont les plus productifs, selon A.G. Nazaryan, les 58 noms suivants sont : main, tête, oeil, coeur, pied, nez, oreille, bouche, dent, bras, peau, doigt, dos, langue, jambe, corps, queue, bee, ventre, cul, patte, gueule , devant, cheveu, poil, figure, epaule, aile, visage, lèvre, visage, talon, gorge, soja, barbe, cornes, ongles, coude, pouce, poing, genou, gosier, menton, côte, fesse, cheville, joue , mâchoire, griffe, moustache, cuisse, poignet, paupière, sourcil, cil, poitrine, crâne, narine (Nazaryan, 1968).

\section{Conclusion}

Parmi les unités phraséologiques, un groupe spécial se distingue par les unités phraséologiques-somatismes, dont la particularité est la présence dans leur structure en tant que composant significatif d'un lexème désignant une partie du corps d'une personne ou d'un animal.

Toutes les unités-somatismes phraséologiques sont divisées en équivalents complets, partiels et non équivalents, en fonction de leur structure lexicale et sémantique.

Cinq composantes des unités-somatismes phraséologiques françaises : tête, œil, cœur, main, bras, pied, jambe, sont les plus courantes.

Selon les caractéristiques sémantiques, dix groupes lexicaux et sémantiques de somatismes sont distingués: caractère, condition physique, état de sentiment, attitude de sentiment, caractéristiques qualitatives d'une personne, activité mentale, portrait, actions et actes d'une personne, statut social, degré de distance de l'objet. Les groupes lexicaux et sémantiques sélectionnés permettent de différencier à la fois les traits généraux inhérents à l'ensemble de la communauté linguistique, et les traits spécifiques des unités phraséologiques-somatismes.

La signification théorique de l'œuvre. Cet ouvrage se distingue des travaux antérieurs consacrés à l'étude des unités phraséologiques, les somatismes, en ce qu'il apporte une certaine contribution à l'étude typologique des langues et contribue au développement des relations interlingues entre porteurs de cultures différentes.

La valeur pratique de l'étude est déterminée par la possibilité d'utiliser les résultats obtenus en phraséographie pour clarifier les définitions du vocabulaire, lors de l'élaboration de dictionnaires phraséologiques bilingues, dans la pratique de la traduction, comme matériel pour des cours particuliers de phraséologie, dans la 
pratique de l'enseignement du français, dans la développement de disciplines particulières, en particulier la typologie et la stylistique comparées.

L'hypothèse de recherche est que la base de la non-équivalence et de l'équivalence incomplète dans différentes langues est une image phraséologique, qui est une composante nationale-culturelle de la sémantique des unités phraséologiques.

Les résultats de la recherche ont été discutés lors de réunions du Département de philologie française et de conférences scientifiques et pratiques.

Le matériau de la recherche était les données d'un dictionnaire étymologique, phraséologique et explicatif unique et bilingue. Pour observer la mise en œuvre des sens des somatismes dans la parole, des exemples d'utilisation des somatismes ont été tirés, choisis parmi les œuvres de fiction d'écrivains français (1100 unités).

L'image phraséologique est une composante essentielle du sens phraséologique. La différence dans la structure figurative est une composante nationale-culturelle dans la sémantique des unités phraséologiques, ce qui indique que chaque langue a des somatismes qui n'ont pas de correspondances phraséologiques dans une autre. L'uniformité est une confirmation des normes culturelles, morales et comportementales communes des locuteurs des langues comparées.

\section{Bibliographic references}

ARSENTIEVA, E.F. 1989. Analyse comparative d'unités phraséologiques, stylistiquement orientées vers une personne, en langues russe et anglaise et problèmes de création d'un dictionnaire phraséologique russe-anglais. Kazan : KSU, 126 p.

BALLY, S. 1955. Linguistique générale et questions de la langue française. Moscow: Maison d'édition de littérature étrangère,

BONNARD, H. 1981. Procedes annexes d'expression: stylistique, theorique, poetique. Paris: Magnard, $241 \mathrm{p}$.

BRUNOT, F. 1927. La pensee et la langue. Paris, 155 p.

COYAUD, M. 1974. Metaphores japonaises dans la faune et flora. In : Semiotica, vol. 11, n.2.

CRESSOT, M. 1947. Le style et ses techniques. Paris, 186 p.

DAUZAT, A. 1945. Traite d'antroponimie franchise. Les noms de famille de France. Paris, $187 \mathrm{p}$.

DAUZAT, A. 1952. Le genie de la langue. Paris, 245 p.

DUBOIS, J. 1965. Grammaire structural: nom et pronom. Paris, $350 \mathrm{p}$.

FAVRET-SAADA, J.1977. Les Mots, la Mort, les Sorts. Paris: Gallimard, 95 p.

FRANCOIS, F. 1968. Le langage et ses fonctions. Le langage: Volume publie sous la direction d'Andre Martinet. Paris: Gallimard, pp.13-19.

GAMILLSCHEG, F. 1957. HistorischefranzosischeSyntaxeTexte. Tubingen, 230 p.

GET, R. 1935. Le Bidois Syntaxe du franfais moderne. Paris,

GRAMMONT, M. 1946. Traite de phonetique. Paris: Librai-rie Delagrave, 480 p.

GREIMAS, A.J. 1966. Semantique structural (Recherche de methode). Paris: Larousse, $96 \mathrm{p}$.

GUIRAUD, P. 1967. Les locutions franchises. 3eme ed. Paris: Presse Universitaire de France, $122 \mathrm{p}$.

JOUET, J. 1990. Les mots du corps. Paris: Larousse, 251 p.

KIRILLOVA, N.N. 2000. Problèmes de phraséologie idio-ethnique. Dokl. int. séminaire. 10-11 novembre 1994. éd. N.N. Kirillova; A grandi. Etat péd. un-t eux. I.A. Herzen ; $\mathrm{SPb}$ : Maison d'édition de l'Université pédagogique d'État russe nommée d'après A.I. Herzen, n. 3.

MARTEL, L. 1883. Petit recueil des proverbes francais. 10-e ed. Paris: Gamier, 329 p. 
MARTIN, C. - COOPER, A. 1974. Mathematical Model of Historical Semantics and the Grouping of Word Meanings into Concepts. Toulouse: Universite de Toulouse, $160 \mathrm{p}$.

MEILLET, A. 1921. Linguistique historique generate Paris, $154 \mathrm{p}$.

MORDKOVITCH, E.M. 1970. Unités phraséologiques somatiques de la langue biélorusse qui n'ont pas d'équivalent dans le texte russe. Questions de philologie russe : résumés de rapports. IVeconférencescientifique et théorique. Samarcande, $235 \mathrm{p}$.

MORDKOVITCH, E.M. 1971. Groupes sémantiques et thématiques d'unités phraséologiques somatiques : problèmes réels de phraséologie Texte. Novosibirsk, $260 \mathrm{p}$.

NAZARYAN, A.G. 1968. Pourquoi parlent-ils le texte français. Moscow, 332 p.

NAZARYAN, A.G. 1972. A propos de l'indépendance sémantique des composants PU dans le texte français. Questions de sémantique des langues PU, slaves, germaniques et romanes. Novgorod, n. 2.

NAZARYAN, A.G. 1978. Texte d'expressions idiomatiques françaises. Moscow. : Éducation,

SHANSKY, N.M. 1969 Phraséologie de la langue russe moderne Texte. Moscow, n. 2 SHANSKY, N.M. 1985. Phraséologie de la langue russe moderne Texte. uch. Manuel. Lycée

TELIA, V.N. 1966. Qu'est-ce que la phraséologie Texte. Moscow : Nauka, 86 p.

TELIA, V.N. 1981. Types de sens linguistiques : sens associé d'un mot dans le texte russe. Moscow: Nauka, 269 p.

TELIA, V.N. 1986. Aspect connotatif de la sémantique des unités nominatives. Texte. otv. éd. AA Ufimtseva; Académie des sciences de l'URSS, Institut de linguistique. Moscou In: Nauka, $141 \mathrm{p}$.

TELIA, V.N. 1996. Phraséologie russe, aspects sémantiques, pragmatiques et linguoculturels Texte. Moscow: Shk. languesrus. culture, $284 \mathrm{p}$.

TELIA, V.N. 1999. Phraséologie dans le contexte de la culture Texte. : recueil d'articles. De l'art. otv. éd. V.N. Télia; A grandi. acad. les sciences. Institut de linguistique. Problèmes, le groupe "Général. phraséologie". M. : Yaz. russe culture, $336 \mathrm{p}$.

TELIA, V.N. 2004. Couches culturelles dans les unités phraséologiques et dans les pratiques discursives Texte. : recueil d'articles. De l'art. otv. éd. V.N. Télia; A grandi. acad. les sciences. Institut de linguistique. Moscow : Yaz. Slaves, Culture, 340 p.

TER-MINASOVA, S.G. 2000. Langue et communication interculturelle Texte . manuel. allocation. Moscow :Slovo, $624 \mathrm{p}$.

VAKK, F. 1964. Sur la phraséologie somatique dans la langue littéraire moderne Texte: dis. Cand. philol. Sciences. Tallinn, 159 p.

VAKK, F. 1968. Sur la phraséologie somatique de la langue estonienne. Questions de phraséologie et compilation de dictionnaires phraséologiques. Texte. Bakou, $135 \mathrm{p}$.

VANDRIES, J.1937. Language Text. Moscow, 407 p.

VEDENINA, L.G. 1988. Particularités de la langue française Texte : un guide pédagogique. Moscow :Éducation, $240 \mathrm{p}$.

VEZHBITSKAYA, A. 1996. Langue. Culture. Cognition. Texte par. de l'anglais.Moscow : Dictionnaires russes, 416 p.66.

\section{Words: 4994}

Characters: 33318 (18,51 standard pages)

Prof. Kulyash Duisekova, dr.

L.N. National Eurasian University Gumilyov

Satbayeva 2

010000 Nur-Sultan

Kazakhstan 
Prof. Saule Issabekova

Kazakh Ablai Khan University of International Relations and World Languages Tole bii, 202

050050 Almaty

Kazakhstan

Prof. Aliya Zagidullina, Dr.,

Kazakh Ablai Khan University of International Relations and World Languages

Tole bii, 202

050050 Almaty

Kazakhstan

Assoc. prof. Gulshat Beysembayeva, dr.

Department of Foreign Philology

L.N. Eurasian National University Gumilyov

Satbayeva 2

010000 Nur-Sultan

Kazakhstan

Assoc. prof. Aitkali Bakitovi

Kazakh al-Farabi National University,

Temiryazeva, 72

050050 Almaty

Kazakhstan

nurbek_lunara@mail.ru

PhDr. Peter Žiak, PhD.

free-lance researcher

High-school St. Ursula

Nedbalova 4

81101 Bratislava

Slovakia

pepeziak@gmail.com 